\title{
A FORCING PROOF OF THE KECHRIS-MOSCHOVAKIS CONSTRUCTIBILITY THEOREM
}

\author{
ANDREAS BLASS
}

ABSTRACT. We show, by forcing, that every subset of $\boldsymbol{K}_{1}$ whose codes form a $\Sigma_{2}^{1}$ set of reals must be constructible.

In [1], Kechris and Moschovakis proved the following theorem by a gametheoretic argument and expressed doubt whether it could be proved by the forcing techniques of Solovay [3].

Theorem (Kechris-Moschovakis). Let $A$ be a set of countable ordinals whose codes form a $\Sigma_{2}^{1}$ set of reals. Then $A$ is constructible.

(For details of the coding of ordinals by reals, see [1].)

The purpose of this note is to prove this theorem by forcing.

Let $A$ be as in the hypothesis of the theorem, and let $P$ be a $\Sigma_{2}^{1}$ formula such that, whenever a real $\alpha$ codes an ordinal $\sigma$,

$$
\sigma \in A \longleftrightarrow P(\alpha)
$$

We may suppose, without loss of generality, that the statement

$$
\forall \alpha, \beta[(\alpha \text { codes the same ordinal as } \beta) \wedge P(\beta) \rightarrow P(\alpha)]
$$

is provable in $\mathrm{ZFC}$, for we may, if necessary, replace the given $P(\alpha)$ with the new $\Sigma_{2}^{1}$ formula

$$
\exists \beta[(\alpha \text { codes the same ordinal as } \beta) \wedge P(\beta)] \text {. }
$$

For each countable ordinal $\sigma$, let $C_{\sigma}$ be the set of one-to-one finite partial functions from $\omega$ to $\sigma$. We think of $C_{\sigma}$ as a notion of forcing (see [2]), and we write $\mathbb{H}_{\sigma}$ for the associated (weak) forcing relation. The forcing language contains a name $G_{\sigma}$ for the generic subset of $C_{\sigma}$ and a name

Received by the editors July 20, 1973 and in revised form, November 28, 1973. AMS (MOS) subject classifications (1970). Primary 04A10, 04A15. 
$\gamma_{\sigma}$ for the well-ordering of $\omega$ (or a finite subset of $\omega$ ) of length $\sigma$ induced by the bijection $\bigcup G_{\sigma}$ from $\omega$ (or a finite subset) onto $\sigma$. Thus,

$$
\varnothing \mathbb{H}_{\sigma} G_{\sigma} \text { is a generic (over the ground model } \check{V} \text { ) subset of } \check{C}_{\sigma} \text {, }
$$
and $\gamma_{\sigma}$ is the well-ordering of $\check{\omega}$ (or a finite subset) induced by $\cup G_{\sigma}$; thus $\gamma_{\sigma}$ is a code for $\check{\sigma}$.

It is easy to check that $C_{\sigma}, G_{\sigma}$ and $\gamma_{\sigma}$ are constructible functions of $\sigma$.

Consider a fixed countable ordinal $\sigma$ and a code $\alpha$ for $\sigma$. Let $C^{*}$ be a notion of forcing with respect to which every condition (weakly) forces:

(4) Every element of $\check{C}_{\sigma}$ belongs to some generic (over $\breve{V}$ ) subset of $\check{C}_{\sigma}$. For example, $C_{\sigma}$ itself is such a notion of forcing, but it is perhaps easier to verify (4) if we take $C^{*}$ such that the power of the continuum is collapsed to $\omega$. With respect to any such $C^{*}$, every condition (weakly) forces the content of the following paragraph.

For every generic (over $\breve{V}$ ) subset $G$ of $\check{C}_{\sigma}$, inducing a well-ordering $\gamma_{G}$ of $\check{\omega}$ (or a finite subset) of length $\check{\sigma}$, we have the following chain of equivalences:

$$
\begin{aligned}
\check{\sigma} \in \check{A} & \leftrightarrow \breve{V} \vDash P(\check{\alpha}) & & \text { by }(1), \\
& \leftrightarrow P(\check{\alpha}) & & \text { by Shoenfield's absoluteness theorem, } \\
& \leftrightarrow P\left(\gamma_{G}\right) & & \text { by (2) as both } \check{\alpha} \text { and } \gamma_{G} \text { code } \check{\sigma}, \\
& \leftrightarrow L[G] \vDash P\left(\gamma_{G}\right) & & \text { by Shoenfield again. }
\end{aligned}
$$

As $G$ is generic over $L$ and $\check{\gamma}_{\sigma}$ denotes $\gamma_{G}$ in the usual interpretation of the forcing language in $L[G]$, the last formula in our chain of equivalences is implied by $L \vDash\left(\varnothing H_{\breve{\sigma}} P\left(\check{\gamma}_{\sigma}\right)\right)$. But conversely, if in $L$ the empty condition does not force $P\left(\check{\gamma}_{\sigma}\right)$, then there is a $p \in \check{C}_{\sigma}$ forcing (in $\left.L\right) \neg P\left(\check{\gamma}_{\sigma}\right)$. By (4), this $p$ is in some generic $G$, so, by the chain of equivalences, $\check{\sigma} \notin \check{A}$. We have therefore

$$
\check{\sigma} \in \check{A} \leftrightarrow L \vDash\left(\varnothing H_{\breve{\sigma}} P\left(\check{\gamma}_{\sigma}\right)\right) .
$$

In the formula (5), which is forced with respect to $C^{*}$, all quantifiers are restricted to $L$. Therefore, we have (in the real world)

$$
\sigma \in A \leftrightarrow L \vDash\left(\varnothing H_{\sigma} P\left(\gamma_{\sigma}\right)\right),
$$

from which it immediately follows (since $\sigma$ was arbitrary) that $A \in L$. 


\section{REFERENCES}

1. A. S. Kechris and Y. N. Moschovakis, Two theorems about projective sets, Israel J. Math. 12 (1972), 391-399.

2. J. R. Shoenfield, Unramified forcing, Proc. Sympos. Pure Math., vol. 13, Part I, Amer. Math. Soc., Providence, R. I., 1971, pp. 357-381. MR 43 \#6079.

3. R. M. Solovay, Measurable cardinals and the axiom of determinateness, Lecture notes for Summer Institute on axiomatic set theory, UCLA (1967).

DEPARTMENT OF MATHEMATICS, UNIVERSITY OF MICHIGAN, ANN ARBOR, MICHIGAN 48104 